\title{
DESENVOLVIMENTO DE UM JOGO EDUCACIONAL DE REALIDADE MISTA VOLTADO PARA O ENSINO MÉDICO: UM RELATO DE EXPERIÊNCIA
}

\begin{abstract}
Érica De la Torre Prado Pereira1; https://orcid.org/0000-0002-8128-5751; Bianca Thais Schneider²; https://orcid.org/0000-0002-1910-2651; Carolina Borges Benedetti; https://orcid.org/0000-0002-5211-2579; Luiza Molinari Bottega4; https://orcid.org/0000-0003-4186-6778; Luiza Ventura Penteado da Silva5, https://orcid.org/00000002-5759-0828; Rodrigo Alberton da Silva6, https://orcid.org/0000-0003-2897-6606; Alexandre Lazaretti Zanatta , https://orcid.org/0000-0002-8430-4882; Pablo Santiago8, https://orcid.org/0000-0002-4798-1366
\end{abstract}

\section{FILIAÇÃO}

(1) Universidade de Passo Fundo, Medical Student

(2) Universidade de Passo Fundo, Medical Student

(3) Universidade de Passo Fundo, Medical Student

(4) Universidade de Passo Fundo, Medical Student

(5) Universidade de Passo Fundo, Medical Student

(6) Universidade de Passo Fundo, Medical Student

(7) Universidade de Passo Fundo, Doutor, Professor do departamento de ciências da computação

(8) Universidade de Passo Fundo, Especialista, Professor do departamento de pediatria

\section{AUTOR CORRESPONDENTE}

Érica De la Torre Prado Pereira. E-mail: delatorre.-e@outlook.com. Rua capitão Araújo, 706, Passo Fundo - Rio Grande do Sul, CEP: 99010-200. Acadêmica de Medicina da Universidade de Passo Fundo.

\section{MENSAGENS-CHAVE}

Há poucos artigos na literatura que abordem o processo de criação de um jogo educacional.

O relato é útil como material de estudo para outros alunos que desejem desenvolver jogos educativos.

A experiência demonstra como é construtivo o trabalho conjunto entre estudantes da medicina e outros cursos em prol da educação.

O processo de elaboração do jogo proporcionou interação com tecnologias de ponta.

O projeto inspira novas formas de união entre tecnologia e aprendizado, bem como a modernização da matriz curricular.

\section{RESUMO}

INTRODUÇÃO: As tecnologias estão progressivamente inseridas na medicina, facilitando simulações práticas e o ensino teórico. O "jogo sério" é uma ferramenta tecnológica que pode agregar no ensino médico, aliando o entretenimento dos games com fundamentos educativos. Partindo deste princípio, um grupo de alunos desenvolveu um jogo sério que utiliza a realidade aumentada no ensino da anemia. Este relato tem como objetivo descrever as experiências vividas e os métodos utilizados na criação de um jogo que possua elementos pedagógicos e proporcione interatividade aos alunos, assim como explicitar fragilidades e potencialidades encontradas no desenvolvimento de projetos interdisciplinares, a fim de auxiliar no embasamento para futuras produções semelhantes. RELATO: O projeto iniciou com uma revisão de literatura dos jogos sérios já existentes e sua aplicação no ensino. Alguns alunos cuidaram da parte do roteiro e programação do jogo, outros da parte clínica da anemia e outros estudaram sobre a parte pedagógica e possíveis avaliações do aprendizado. Também, ao final da elaboração do jogo, desenvolveu-se protocolos de testagem com questionários validados e, no momento, o jogo encontra-se 
em fase de testes na disciplina de hematologia. DISCUSSÃO: A experiência evidenciou a importância da interdisciplinaridade na educação médica, já que na maioria das escolas médicas ainda se possui um modelo de educação engessado e pouco criativo. Além disso, possibilitou a introdução de uma nova abordagem educacional e de uma nova tecnologia no modelo tradicional de ensino. CONCLUSÃO: Nosso trabalho serviu para iniciar o contato entre duas áreas distintas dentro da universidade e estimular a produção de novas ferramentas de ensino, agregando ao currículo médico uma nova abordagem pedagógica. Por fim, ao instigar sobre o ensino complementado pela tecnologia, novos jogos estão sendo desenvolvidos seguindo o modelo proposto e publicações puderam ser realizadas a partir das revisões literárias das avaliações pedagógicas e modelos de jogos educacionais.

\title{
PALAVRAS-CHAVE: Educação Médica; Tecnologia educacional; Aprendizado interativo.
}

\begin{abstract}
INTRODUCTION: Technologies are increasingly embedded in medicine, facilitating practical simulations and theoretical teaching. The "serious game" is a technological tool that can add to medical education, combining game entertainment with educational fundamentals. Based on this principle, a group of students developed a serious game that uses augmented reality to teach anemia. This report aims to describe the experience and the methodology used in creating a game that has pedagogical elements and provides interactivity to students, as well as expose weaknesses and possibilities found on the development of interdisciplinary projects in the means of assisting similar future projects. REPORT: The project started with a literature review of serious games that already existed and their application in teaching. Some of the students took care of the script and programming part of the game, others took care of the anemia clinical part and others studied about the pedagogical and possible learning assessments. Also, at the end of the elaboration of the game, testing protocols were developed with validated questionnaires and, at the moment, the game is undergoing tests in the hematology discipline. DISCUSSION: The experience showed the importance of interdisciplinarity in medical education, since most medical schools still have a model of education that is plastered and not very creative. In addition, it enabled the introduction of a new educational approach and of a new technology in the traditional teaching model. CONCLUSION: Our work served to initiate contact between two different areas within the university and to stimulate the production of new teaching tools, adding to the medical curriculum a new pedagogical approach. Lastly, instigating teaching complemented by technological tools, new games are already being developed following the model proposed and publications were made from the literature reviews about pedagogical evaluation $e$ learning game models.
\end{abstract}

\section{KEYWORDS: Medical Education; Educational technology; Interactive learning}

\section{INTRODUÇÃO}

O avanço tecnológico na medicina requer a formação de profissionais que se adaptem rapidamente ao uso de novas ferramentas. Essa tendência inevitável à integralização da Medicina com as novas tecnologias em ascensão, ainda, evidencia a necessidade iminente de se instaurarem grupos de trabalho e de pesquisa interdisciplinares, que associam saúde e tecnologia no desenvolvimento de novas técnicas e metodologias de cuidado. No âmbito da educação, esse aspecto demonstra-se ainda mais relevante, visto que a disseminação do uso de dispositivos digitais para o ensino consiste em uma realidade já presente em nosso meio ${ }^{1}$.

Nesse sentido, já existem tecnologias aplicadas no ensino médico, como aplicativos, laboratórios de simulação, jogos, que objetivam o treinamento de habilidades técnicas. Os serious games (jogos sérios) se destacam dentre estas tecnologias, diferente dos outros jogos, eles transcendem o objetivo de entretenimento, agregando a construção de conhecimentos práticos em sua composição². O uso dos jogos sérios na educação, para Machado et al., permite uma maior capacidade de compreensão e fixação do conteúdo abordado, uma vez que formaliza a união de aspectos lúdicos a aspectos especificamente técnicos.

Apesar de bem difundido, os jogos sérios têm seus benefícios educacionais baseados em suposições, com poucas evidências formais e concretas ${ }^{3}$. Assim, em janeiro de 2019, estudantes de uma faculdade de Medicina iniciaram o desenvolvimento de um projeto piloto de jogo sério voltado para o ensino de anemias, com o objetivo de testar sua eficácia educacional como método complementar às aulas teóricas tradicionais.

Com base nas ferramentas disponíveis e na tentativa de explorar tecnologias mais recentes optou-se pelo uso da realidade aumentada. A realidade mista, ou aumentada, traz elementos da própria realidade com os elementos virtuais, possibilitando juntar objetos físicos reais e objetos virtuais em um mesmo ambiente, também, promove a interação do usuário com estes enriquecendo a experiência ${ }^{4}$.

O objetivo deste relato é descrever o método utilizado para desenvolver um jogo sério dentro da faculdade de medicina, orientando sobre a dinâmica do trabalho em equipe e evidências encontradas nas revisões literárias e na avaliação pedagógica. Além disso, abordar as fragilidades, potencialidades e resultados obtidos durante a elaboração do projeto, a fim de torná-lo reprodutível e aproveitável como ferramenta de embasamento para futuras produções semelhantes.

\section{RELATO}

O projeto em questão reuniu acadêmicos dos cursos de Medicina e de Ciência da Computação na produção de um jogo voltado para o ensino de Hematologia, cumprindo com o objetivo de agregar conhecimentos e habilidades de duas áreas que, apesar de aparentemente distintas, encontram- 
se intensamente fusionadas na atualidade. Durante a execução do jogo, tanto o envolvimento de alunos de Medicina na familiarização com as ferramentas de programação e desenvolvimento do programa, como a aprendizagem dos alunos de Computação a respeito das anemias, possibilitou uma permuta de informações e a formação de um vínculo interdisciplinar que, seguramente, denota o início de uma importante e necessária associação de profissionais e de competências.

Assim, para que o projeto fosse iniciado, foram estabelecidas metas. A curto prazo, os alunos deveriam desenvolver aprendizado sobre os diferentes modelos de jogos existentes (para que pudessem escolher um), buscar relatos sobre programas similares já desenvolvidos, estudar e sistematizar o conteúdo a ser transmitido através do jogo, bem como procurar por um método eficaz de avaliação da eficiência do mesmo no ensino. Já as metas estabelecidas para longo prazo foram a criação do roteiro e dos cenários, a programação e, por fim, sua testagem, a ser aplicada aos acadêmicos de medicina e avaliada por meio de questionários desenvolvidos pelos próprios alunos com base em questionários já validados.

Dessa forma, orientados por dois professores, um hematologista e um cientista da computação, os alunos de medicina foram distribuídos em três grupos, cada um sendo liderado por um monitor voluntário. O primeiro grupo seria responsável por uma revisão de literatura, como forma de buscar embasamento sobre programas já desenvolvidos e a maneira como foram testados. O segundo grupo estudaria diferentes modelos de jogos e desenvolveria os roteiros e cenários e, por último, caberia ao terceiro grupo o estudo aprofundado acerca das anemias, como forma de garantir a qualidade do conteúdo ensinado no jogo.

Entretanto, tendo em vista que os alunos não haviam passado pela cadeira de hematologia, possuíam pouca experiência com o desenvolvimento de programas e, ainda, a divisão em grupos poderia causar disparidade entre os conhecimentos adquiridos, alguns desafios precisaram ser vencidos. Para isso, foram organizados encontros semanais em que o professor hematologista e os alunos do terceiro grupo lecionaram aulas sobre anemia. Já no mês de abril de 2019, todos os alunos visitaram o Parque Tecnológico da UPF, onde conheceram o acadêmico e a mestranda de Tecnologia da Informação, responsáveis pela programação do jogo. Nesse local, os estudantes puderam conhecer e testar as tecnologias de Realidade Virtual e Realidade Ampliada, sendo que a segunda foi escolhida para ser aplicada ao jogo, o qual seria praticado por meio de óculos tecnológico.

Em paralelo, os alunos do primeiro grupo analisaram 20 artigos, guiados pelos termos de busca ((mixed OR augmented) AND reality AND game AND (teaching OR learning OR education OR training) AND medicine), dos quais 9 foram selecionados e resumidos. Os alunos do segundo grupo decidiram o cenário e a história do jogo: um passeio pelos diferentes órgãos envolvidos na fisiopatologia da anemia, em que cada órgão oferece determinadas informações necessárias para o preenchimento do hemograma e realização do diagnóstico.

Por fim, os alunos do terceiro grupo produziram um compilado abordando as diferentes anemias existentes, bem como selecionaram o conteúdo de "anemia ferropriva" para ser abordado no jogo - decisão baseada na alta prevalência da patologia e, portanto, da importância do seu reconhecimento pelo médico clínico.

A partir dos estudos feitos, especialmente com relação aos modelos de jogos semelhantes, os alunos puderam elaborar uma revisão sistemática sobre os resultados obtidos nas experiências prévias com educação através de jogos. Observaram-se prometedores efeitos a partir dessas experiências anteriores, sobretudo no âmbito de habilidades interpessoais como resolução de problemas, pensamento criativo e manejo do tempo, além da fixação do conhecimento teórico propriamente dito. Essa revisão gerou um trabalho apresentado pelos acadêmicos da pesquisa em eventos locais, nacionais e até internacionais na área de novas tecnologias e educação.

Um importante problema encontrado durante o projeto foi 0 método de avaliação do jogo educacional, o qual por vezes não é capaz de mensurar os conhecimentos obtidos pelos alunos. A fim de buscar a melhor forma de análise, o primeiro grupo incluiu na revisão de literatura a busca por questionários validados que avaliassem a retenção de conhecimento após uma atividade de ensino. Ao final, com base nessa pesquisa, os estudantes desenvolveram instrumentos avaliativos por meio de uma metodologia adaptada, baseada nas etapas do processo de construção de medidas de avaliação em Treinamento, Desenvolvimento e Educação, apresentado por Mourão e Menezes ${ }^{5}$.

Os questionários foram divididos e organizados em categorias avaliativas, em níveis de reação e aprendizagem, de Kirkpatrick ${ }^{3}$. As técnicas de coleta de dados foram divididas em comunicação por meio de questionários (incluindo pré e pós-testes) e observação humana (observação e registro de informações, pela equipe de desenvolvimento, padronizado por roteiro). Nos questionários foi usada a escala do tipo Likert, de 1 (discordo fortemente) a 5 (concordo fortemente), além de questões abertas e de múltipla escolha6. Ademais, visando uma aplicação padronizada, foi elaborado um protocolo de avaliação. Esse protocolo organizou os questionários em: perfil do jogador, pré e pós-testes (nível de aprendizagem) e reação ao jogo (nível de reação ${ }^{5}$.

A respeito da realização desta atividade, podem-se notabilizar distintos aspectos positivos, desde a interdisciplinaridade e aproximação de acadêmicos de diferentes áreas, até 0 aprofundamento no conhecimento de tema essencial e preeminente na prática clínica da medicina. Ademais, o desenvolvimento de um modelo que poderá ser aproveitado futuramente para a expansão de novos projetos e assuntos consiste em um benefício que se estende aos demais acadêmicos não participantes do projeto de pesquisa. Como aspectos negativos, por outro lado, observa-se a dificuldade no compartilhamento do conhecimento entre as distintas áreas 
que, apesar de ser um dos objetivos principais do projeto, acabou consumindo considerável tempo além daquele já previsto para a elaboração do jogo. Recomenda-se que equipes subsequentes à realizarem projetos similares tenham consciência da demanda de tempo da empreitada, bem como da importância de se ter um contato próximo e acompanhamento durante todo o processo.

Em geral, tem-se agora um "projeto-piloto" já desenvolvido e pronto para ser testado. A proposta da aplicação e da realização dos testes com humanas foi apreciada e aprovada pelo Comitê de Ética da Universidade XXXXX e submetida à Plataforma Brasil sob o CAAE de $\mathrm{n}^{0}$ $X X X X . X . X X X . X X X$. Espera-se que, após a análise dos dados obtidos pela aplicação dos questionários, seja comprovada a eficiência da utilização do Jogo Sério como ferramenta didática auxiliar ao professor. É esperado também que o jogo apresentado sirva de modelo para a criação de novos jogos didáticos abordando outros conteúdos de hematologia e possivelmente, outras

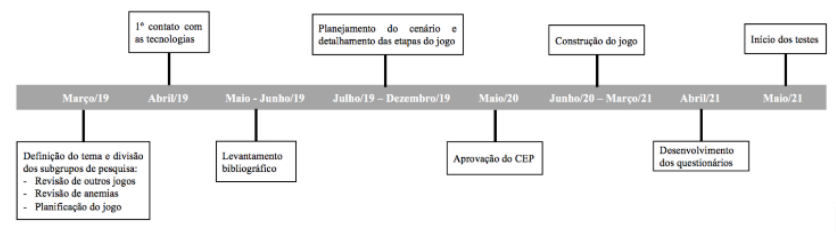

Figura 1 - Linha Temporal do Projeto Fonte Própria

\section{DISCUSSÃO}

A experiência de desenvolver um jogo educativo digital evidenciou duas necessidades no que tange a educação médica: desenvolver uma matriz curricular que transcenda o campo da medicina e proporcione atividades interprofissionais; e introduzir nessa matriz projetos que gerem contato entre os estudantes de medicina e as tecnologias que os futuros médicos encontraram em suas profissões. Desenvolver competências profissionais de comunicação, interação e colaboração profissional gera o aprimoramento da formação e também da atenção em saúde 7 .

O desafio de desenvolver esse "jogo sério" em conjunto com alunos e professores da Ciência da Computação expandiu os horizontes dos alunos em todos os sentidos. Infelizmente, a graduação médica brasileira ainda tem forte influência do modelo flexneriano e, por isso, quando desenvolvemos projetos e ideias fora do ambiente engessado das escolas de medicina, essas experiências sempre se mostram enriquecedoras e estimulantes para nossa criatividade ${ }^{8}$.

Segundo as Diretrizes Curriculares Nacionais dos Cursos de Graduação em Medicina, uma das habilidades esperadas dos egressos envolve aprender a aprender. Assim, a estrutura curricular deve contemplar uma organização centrada no estudante, capacitando-o a manusear os recursos tecnológicos existentes em favor de sua formação e atualização, trazendo uma oportunidade de incluir as Tecnologias Digitais de Informação e Comunicação (TDICs) como parte de uma nova concepção de ensino e aprendizagem9. Dessa forma, o projeto também evidenciou a importância de se instituírem aulas, módulos, eventos e outras formas de contato dos acadêmicos com as ferramentas tecnológicas que nos cercam e que estão sendo desenvolvidas. No atual mercado de trabalho, e ainda mais no futuro, o médico precisará utilizar desses instrumentos para ter competitividade entre os pares e oferecer o melhor cuidado possível aos seus pacientes. Da mesma forma, a aproximação interdisciplinar ocorrida durante a realização deste projeto antevê um aspecto fundamental das contemporâneas relações de trabalho no meio de saúde, em que o bom convívio e sincronia entre os profissionais de diferentes áreas configura condição fundamental para a qualidade do serviço.

Outros trabalhos também descrevem experiências e podem ser utilizados como embasamento para a criação de outros jogos sérios ${ }^{10,11}$. Além disso, nosso trabalho foi o precursor para o desenvolvimento de outros jogos na universidade, 0 que oportunizará novas abordagens tecnológicas e pedagógicas. A expansão desse modelo de metodologia de ensino para o aprendizado de outras áreas da medicina, adaptada para suas próprias particularidades e requisitos, caracterizaria um importante avanço no uso dessas tecnologias, o que poderia agregar nas evidências da real eficácia dos jogos sérios além de implementar na qualificação do ensino médico.

Como todo projeto, percebemos algumas fragilidades e pontos que podem ser melhor desenvolvidos no futuro. Ressaltamos a importância criar equipes multiprofissionais que envolvam áreas além da computação e medicina, a fim de ampliar e aprimorar a experiência e o aprendizado. Também, é fundamental, durante o planejamento, a compreensão de que se trata de um processo com diversas etapas e duração prolongada. Além disso, em projetos locais precursores dessa modalidade de ensino e aprendizagem, é fundamental diagnosticar previamente as barreiras que serão enfrentadas para a inserção do jogo na rotina curricular.

\section{CONCLUSÃO}

Portanto, a experiência foi muito educativa e integrou conhecimento das duas áreas, medicina e informática, o próximo passo é a testagem do jogo com o intuito de analisar se o mesmo atenderá ao requisito de ensino na hematologia. No entanto, tem-se como fator limitante que o jogo foi pautado somente na área de hematologia e com apenas um caso clínico. Caso comprovada a sua eficiência, poderá ser inserido como instrumento de aprendizado desta disciplina. Já estão sendo encaminhados outros jogos para serem produzidos, utilizando este como modelo. Em vista disso, evidencia-se que esse trabalho refletiu em um maior contato dos acadêmicos com as ferramentas tecnológicas, produziram revisões que puderam ser publicadas e que os acadêmicos poderão contar com jogos na aprendizagem em um futuro próximo. Afinal, deve-se levar em consideração que, no mercado de trabalho atual e no futuro, o médico deverá saber utilizar essas ferramentas para oferecer o melhor cuidado possível aos seus pacientes. 


\section{CONFLITO DE INTERESSE}

Os autores declaram que não há nenhum conflito de interesse presente neste artigo.

\section{FINANCIAMENTO}

Os autores declaram que não houve fontes de financiamento.

\section{REFERÊNCIAS}

1. Pereira TA, Areco KCN, Tarcia RML, Sigulem D. Uso das Tecnologias de Informação e Comunicação por Professores da Área da Saúde da Universidade Federal de São Paulo. Rev. bras. educ. med. 2016 Mar;40(1):59-66.

Disponível em: http://www.scielo.br/scielo.php?script=sci arttext\&pid=S $\underline{0100-55022016000100059 \& \operatorname{lng}=\text { en\&nrm=iso }}$

2. Machado LS, Moraes RM, Nunes FLS, Costa RMEM. Serious games baseados em realidade virtual para educação médica. Revista Brasileira de Educação Médica. 2011 Jun;35(2):254-62. Disponível em: http://www.scielo.br/scielo.php?script=sci arttext\&pid=s $\underline{0100-55022011000200015}$

3. Savi $R$, Wangenheim CG, Ulbricht V, Vanzin $T$. Proposta de um Modelo de Avaliação de Jogos Educacionais. Revista Novas Tecnologias na Educação. 2010 Dez; 8(3). Disponível em: https://seer.ufrgs.br/renote/article/view/18043/10630

4. Lopes LMD, Vidotto KNS, Pozzebon E, Ferenhof HA. Inovações educacionais com o uso da realidade aumentada: uma revisão sistemática. Educ. Rev. 2019 Mar;35:e197403.

5. Abbad GS, Mourão L, Meneses PPM, Zerbini T, Borges-Andrade JE, Vilas-Boas R. Medidas de Avaliação em treinamento, desenvolvimento e educação: ferramentas para gestão de pessoas [dissertação]. [Porto Alegre (BR)]: Artemed; 2012. 50$63 \mathrm{p}$.

6. Moura Jr. AF, Vieira FMS, Lacerda MM. Reflexões sobre Avaliação de Games Educacionais. Seminário Jogos Eletrônicos, Educação e Comunicação, 12. 2017, Salvador. Anais: 2017. p 11 - 20. Disponível em: https://revistas.uneb.br/index.php/sjec/article/view/3547

7. Freire, José Rodrigues et al. Educação Interprofissional nas políticas de reorientação da formação profissional em saúde no Brasil. Saúde em Debate [online]. 2019, v. 43, n. spe1 [Acessado 9 Julho 2021] , pp. 86-96. Disponível em: <https://doi.org/10.1590/010311042019S107>. Epub 16 Set 2019. ISSN 2358-2898. https://doi.org/10.1590/0103-11042019S107.
8. Ferreira IG, Carreira LB, Botelho NM. Mobilidade internacional na graduação em medicina: relato de experiência. ABCS Health Sci. 2017 Feb;42(2):115-19. Disponível em: https://www.portalnepas.org.br/abcshs/article/view/1013

9. Gorgens, PRC, Andrade, PCR. Educação médica e tecnologias digitais de informação e comunicação: possibilidades e dilemas. Revista Médica de Minas Gerais. 2018; 28(41):1-7. Http://dx.doi.org/10.5935/22383182.20180154

10. Conceição, KR, Oliveira, MA. Desenvolvimento de um Jogo Sério para o ensino de Anatomia do membro inferior do Esqueleto Humano. 69 f. TCC (Graduação) - Curso de Tecnologias da Informação e Comunicação, Universidade Federal de Santa Catarina, Araranguá, 2016.

11. Santo, A. Um relato sobre a capacitação e a produção de um jogo sério: a experiência de um projeto de extensão com um game para conscientização da adoção de animais. XVIII SBGAMES; 2019, Rio de Janeiro. 